\title{
Caractéristiques physico-chimiques, phytochimiques et toxicité des espèces végétales utilisées comme emballages alimentaires en Afrique de l'Ouest
}

\author{
Caroline F. ONZO ${ }^{1,2}$, Paulin AZOKPOTA ${ }^{1,2^{*}}$, Pierre AGBANI ${ }^{3}$, Fernand GBAGUIDI ${ }^{4}$, \\ Joseph D. HOUNHOUIGAN ${ }^{1,2}$ et Dansou KOSSOU ${ }^{5}$ \\ ${ }^{I}$ Laboratoire de Formulations Alimentaires et de Biologie Moléculaire (LAFAB) ; Département de Nutrition, \\ Sciences et Technologie Alimentaires, Faculté des Sciences Agronomiques, Université d'Abomey-Calavi, \\ 01 BP 526, Cotonou, Bénin. \\ ${ }^{2}$ Laboratoire de Biochimie Microbienne et de Biotechnologie Alimentaire ; Département de Nutrition, \\ Sciences et Technologie Alimentaires ; Faculté des Sciences Agronomiques, Université d'Abomey-Calavi, \\ 01 BP 526, Cotonou, Bénin. \\ ${ }^{3}$ Laboratoire d'Ecologie Appliquée, Département d'Aménagement et de Gestion des Ressources Naturelles ; \\ Faculté des Sciences Agronomiques, Université d'Abomey-Calavi ; 01 BP 526, Cotonou, Bénin. \\ ${ }^{4}$ Laboratoire de Pharmacognosie et des Huiles Essentielles (L.A.P.H.E) ; Faculté des Sciences et techniques, \\ Université d'Abomey-Calavi, 01 BP 526, Cotonou, Bénin. \\ ${ }^{5}$ Département de Production Végétale, Faculté des Sciences Agronomiques, Université d'Abomey-Calavi ; \\ 01 BP 526, Cotonou, Bénin. \\ *Auteur correspondant, E-mail : azokpotap@yahoo.fr ; paulin.azokpota@fsa.uac.bj
}

\section{RESUME}

Le présent travail vise à caractériser quatre espèces de feuilles végétales utilisées comme emballages alimentaires en Afrique de l'Ouest. La caractérisation a été réalisée au plan physico-chimique, phytochimique et de la toxicité au moyen de méthodes de références. Il est apparu que les teneurs en protéines, en lipides totaux et en cendres totales sont respectivement de 6,$0 ; 4,6 ; 8,7 \%$ pour Tectona grandis $; 16,0 ; 4,3 ; 9,1 \%$ pour Musa sapientum; 12,$5 ; 4,6 ; 7,0 \%$ pour Thalia geniculata et 13,4; 2,0; 7,2\% pour Manihot esculenta. Une diminution significative de ces valeurs a été observée après 3 jours de conservation des produits emballés dans ces espèces de feuilles. Le fer est présent dans toutes les espèces, notamment dans Manihot esculenta $(8,4 \%)$, Tectona grandis $(4,4 \%)$ avec une légère diminution après emballage des produits. La teneur en vitamine $\mathrm{C}$ est présente uniquement dans les feuilles de Manihot esculenta dans l'ordre de 0,4\%. Les tanins, les catéchiques, les anthocyanes, les leuco-anthocyanes, les mucilages, les flavonoïdes et les coumarines ont été identifiés dans toutes les espèces de feuilles analysées. Toutes les espèces de feuilles analysées se sont révélées non toxiques.

(C) 2014 International Formulae Group. All rights reserved.

Mots clés : Emballages végétaux, emballages alimentaires, Bénin, valeur nutritionnelle, toxicité des plantes, caractéristiques phytochimiques.

\section{INTRODUCTION}

Les emballages alimentaires jouent un grand rôle dans la sécurisation et la protection des denrées alimentaires incluant les matières premières et les produits issus des différentes transformations agroalimentaires (Cavassilas, 2006). Au plan technique, les emballages assurent la protection, le transport et la conservation des aliments tout en étant un symbole de publicité pour les industries 
agroalimentaires (Karel et Heidelbaugh, 1975 ; Pothet, 2003). Les éléments constitutifs d'un emballage sont parfois des absorbants ou humectants (d'oxygène ou d'humidité), des précurseurs d'arôme ou de substances antimicrobiennes, de conservateurs ou d'améliorants. Au-delà de son rôle technique, l'emballage est une marque d'authenticité, d'originalité de l'aliment, de l'entreprise productrice, de sa propriété ou du pays producteur des biens. L'urbanisation croissante de la population et le développement des filières agroalimentaires dans le monde ont conduit à un accroissement rapide de la demande en emballages alimentaires, notamment dans les pays en développement (Hounhouigan, 2000).

Dans le monde, il existe une diversité d'emballages utilisés dans le domaine agroalimentaire. Au cours des dix dernières décennies, on a assisté à une prolifération d'emballages plastiques, principalement dans les industries agroalimentaires qui en absorbent environ $65 \%$ contre environ $35 \%$ pour les autres secteurs (AFNOR, 1986; Reyne, 1991).

En Afrique, on a assisté, ces dernières années, à un regain d'emballages plastiques utilisés pour de nombreux aliments, notamment dans les pays de l'Afrique subsaharienne y compris le Bénin qui est situé entre le Nigéria et le Ghana, deux grands pays importateurs d'emballages plastiques (Codjia, 2000). S'il est vrai que les emballages plastiques sont très pratiques, peu encombrants, très esthétiques et particulièrement adaptés au pouvoir d'achat des populations pour la plupart à faibles revenus dans ces pays, il est aussi évident qu'une fois utilisés, ces emballages non biodégradables, deviennent très encombrants dans la nature, posant ainsi de graves problèmes de pollution environnementale difficilement gérables par ces pays à ressources limitées (Kazazian, 1995 ; Adejumo et Ola, 2008). Par ailleurs, la pratique qui consiste à emballer des aliments chauds prêts à être consommés dans les emballages plastiques est courante, ce qui engendre des risques de contaminations chimiques dont les effets peuvent être néfastes à court ou à long terme. En effet, bon nombre d'additifs de fabrication et de monomères résiduels plastiques ayant un impact négatif sur la santé du consommateur et la qualité du produit peuvent migrer vers les aliments emballés, notamment les produits liquides et gras (Reyne, 1991 ; Adegunloye et al., 2006).

De nos jours, les consommateurs sont de plus en plus réticents à conditionner les denrées alimentaires dans des emballages en plastiques. En revanche, certaines espèces de feuilles végétales sont utilisées de tradition depuis de vieilles dates, voire des dates séculaires comme emballages alimentaires biodégradables. Par ailleurs, ces espèces de feuilles constituent donc des alternatives à l'utilisation des sachets en plastiques, principalement du fait des nombreux avantages naturels que ces feuilles offrent. En effet, ces feuilles sont formées en surface d'une couche de cire et de matériaux divers assurant leur imperméabilité à l'eau (nervures, tissus plus riches en eau) leur donnant à la fois la souplesse et la résistance mécanique requises au conditionnement. De plus, ces feuilles contiendraient des composés actifsaromatiques, des colorants, des enzymes (papaïne par exemple) qui migreraient de la feuille végétale vers le produit alimentaire au cours de la conservation du produit. De ce fait, les feuilles végétales présenteraient des avantages comparatifs par rapport aux emballages plastiques non biodégradables.

L'utilisation des feuilles végétales comme emballages alimentaires est déjà intégrée dans l'artisanat agroalimentaire au Bénin depuis longtemps. En effet, le Bénin dispose de nombreuses potentialités en matière d'emballages végétaux déjà d'utilisation courante (Boko et al., 1997). Le développement économique et ses conséquences ont entraîné le transfert de ces emballages végétaux en milieux urbains où ils 
se trouvent en compétition permanente avec les emballages en plastiques importés ou de fabrication locale (Hounhouigan, 2000). Ainsi, de nombreuses espèces végétales utilisées comme emballages, principalement au Centre et au Sud du Bénin ont été recensées (Onzo, et al., 2013). Certaines espèces de feuilles végétales sont utilisées à la fois comme emballages alimentaires et consommées comme de légumes feuilles pour différentes préparations culinaires, notamment les sauces-légumes. C'est le cas de Manihot esculenta, Manihot glaziovii, Sterculia tragacantha, Triplochiton scleroxylon par exemple (Onzo et al., 2013).

Eu égard à tout ce qui précède, il est indispensable de promouvoir et de valoriser ces espèces de feuilles végétales utilisées comme emballages alimentaires. Cependant, pour une meilleure valorisation de ces feuilles comme emballages, beaucoup de questions encore sans réponses méritent d'être élucidées. Il s'agit, par exemple, de leur innocuité. En effet, certaines espèces de feuilles végétales utilisées comme emballages sont potentiellement riches en facteurs antinutritionnels (Grubben, 1975) et les prétraitements qu'elles subissent avant l'utilisation ne garantissent toujours pas l'innocuité requise (Onzo et al., 2013). Cet aspect est d'autant plus préoccupant pour les espèces végétales qui sont à la fois utilisées comme emballages et consommées comme légumes feuilles. Pour les mêmes raisons, l'évaluation des substances nutritionnelles (encore non connues à ce jour) sera d'une importance capitale dans la formulation des régimes alimentaires. En outre, les composés aromatiques potentiels au niveau de certaines espèces pourraient être extraits pour leur incorporation dans des recettes culinaires. Certaines espèces végétales utilisées comme emballages alimentaires contiendraient des composés phytochimiques dont certains éléments peuvent être exploités comme des antioxydants. Des composés d'autres espèces contiendraient des facteurs antinutritionnels qui rendraient non disponibles certains minéraux dans l'organisme. Toutes ces préoccupations constituent des questions de recherche qui sont abordées par le présent travail dont les objectifs spécifiques visent à : (i)- déterminer les éléments nutritifs et les composés phytochimiques dans les espèces de feuilles végétales couramment utilisées comme emballages dans l'artisanat agroalimentaire au Bénin ;

(ii) - évaluer la toxicité desdites feuilles.

\section{MATERIEL ET METHODES \\ Caractérisation physico-chimique des espèces de feuilles végétales ciblées \\ Echantillonnage des espèces}

Les espèces de feuilles végétales qui ont fait l'objet de la présente étude ont été sélectionnées parmi les espèces végétales précédemment identifiées comme servant d'emballages alimentaires au Sud et au Centre du Bénin (Onzo et al., 2013). En outre, les critères ci-après ont été utilisés pour la sélection :

- espèces utilisées à la fois au Sud et au Centre du Bénin ;

- espèces utilisées à la fois comme emballages et légume-feuilles ;

- espèces utilisées comme emballages pour leurs vertus technologiques ou thérapeutiques selon les productrices.

Sur cette base, quatre (4) espèces de feuilles végétales utilisées comme emballages végétaux ont été retenues. Il s'agit de: Tectona grandis, Musa sapientum, Thalia geniculata utilisées pour emballer l'akassa (appellation du produit par l'ethnie Goun au Bénin) et Manihot esculenta pour le lio (appellation par l'ethnie Fon au Bénin). L'akassa est une pâte fermentée à base de maïs cuite à la vapeur après avoir été emballée dans les feuilles. Le lio est également une pâte légèrement fermentée cuite à la vapeur mais plus compacte. 


\section{Prétraitement des produits emballés et des emballages-feuilles}

Les deux produits emballés dans les espèces sélectionnées sont conservés pendant trois (3) jours à température ambiante (25-30 ${ }^{\circ} \mathrm{C}$ ), le temps d'un probable transfert d'éléments des feuilles vers les produits emballés et vise versa. La dernière couche de feuilles directement en contact avec le produit emballé a servi d'échantillon pour les analyses. Les feuilles ont subi différents traitements en fonction de leur nature et du produit emballé, conformément aux pratiques des productrices comme précédemment rapporté (Onzo et al., 2013).

Détermination de la teneur en eau

La teneur en eau des feuilles a été déterminée suivant la méthode A.O.A.C (1984) (44-15A).

Dosage des constituants chimiques et nutritifs

$>\quad$ Protéines: La teneur en protéines totales des feuilles a été dosée par la méthode du biuret (Gornall et al., 1949). Le principe du dosage se repose sur le fait qu'en milieu alcalin, les composés contenant au moins deux groupements amides voisins forment avec les ions cuivriques $\mathrm{Cu}^{2+}$ un complexe bleu violet qui est dosé par spectrophotométrie à $540 \mathrm{~nm}$. L'expérimentation a été réalisée en deux étapes. Dans une première étape, les solutions d'étalonnage ont été préparées et réparties dans six tubes à essais comme indiqué dans le Tableau 1. Dans une deuxième étape (phase de test), deux des 6 tubes (tubes 6 à 7) ont été remplis avec la solution protéique de feuilles séchées à analyser (Tableau 2).

Les solutions tests ont été obtenues par centrifugation (3000 tr/min) du mélange de 5 $\mathrm{g}$ de poudre de feuilles séchées dans de l'eau distillée, pendant 10 minutes à trois reprises. Le surnageant a été recueilli et quantifié. La troisième étape a consisté au dosage proprement parlé. L'expérience a été faite en double. Les tubes ont été laissés pendant 30 minutes à l'obscurité à la température ambiante, et la D.O. (densité optique) à 540 $\mathrm{nm}$ a été lue à l'aide d'un spectrophotomètre de type Biomate ${ }^{\mathrm{TM}}$ 3. A l'aide de la courbe d'étalonnage, le pourcentage des protéines brutes contenues dans chaque échantillon a été calculé utilisant la formule suivante :

$\%$ Protéines $=\frac{8.10^{-4} \times \mathrm{V} \times \mathrm{q}}{\mathrm{m}}(\mathrm{g} / 100 \mathrm{~g})$

Avec: q : Concentration massique de protéines dans l'échantillon $(\mathrm{g} / \mathrm{ml}) ; \mathrm{V}$ : Volume total de filtrat après centrifugation $(\mathrm{ml}) ; \mathrm{m}$ : prise d'essai $(\mathrm{g})$

$>\quad$ Lipides totaux : Les lipides totaux ont été déterminés par la méthode d'extraction au Soxhlet. C'est une méthode d'extraction par percolation au cours de laquelle le solvant, sous l'effet de la chaleur, est distillé et condensé.

Cendres totales : Dix grammes (10 g) de chaque échantillon de feuilles ont été introduits dans des boîtes de porcelaine préalablement séchées et pesées. Cet ensemble a été mis au four à $550{ }^{\circ} \mathrm{C}$ pendant 24 heures et pesé de nouveau après refroidissement. Le taux de cendre brute de chaque échantillon à été calculé par la formule suivante, à savoir :

Taux de cendre $(\%)=\frac{P_{f}-P_{o}}{P_{e}}$

Avec $: \mathrm{P}_{\mathrm{f}}=$ Poids final (boîte + échantillon) après incinération; $\mathrm{P}_{\mathrm{e}}=$ Poids d'échantillon pesé $; \mathrm{P}_{\mathrm{o}}=$ Poids des boîtes vides

$>\quad$ Dosage du Fer : Le fer a été dosé par colorimétrie. Le principe de dosage repose sur le fait que le réactif pour le fer (Ferrover) utilisé à cet effet convertit la grande partie du fer insoluble $(\mathrm{Fe})$ de l'échantillon en fer ferreux $(\mathrm{Fe})$ soluble. Le fer ferreux réagit avec la 1-10 phénanthroline (indicateur) du réactif pour développer une coloration orange proportionnelle à la concentration de fer. $\mathrm{La}$ lecture est obtenue à $510 \mathrm{~nm}$ au spectrophotomètre d'absorption moléculaire.

> Dosage de la Vitamine C. La vitamine C a été dosée par le densitomètre Camag TLC Scanner III. La solution standard de la vitamine $\mathrm{C}$ a été préparée en dissolvant $5 \mathrm{mg}$ de l'acide ascorbique standard dans $5 \mathrm{ml}$ d'eau distillée. Environ $5 \mathrm{~g}$ de feuilles fraiches bien pilées dans un mortier en porcelaine ont 
été pesés. A la masse obtenue, $10 \mathrm{ml}$ d'eau distillée ont été ajoutés. A $2 \mathrm{ml}$ du filtrat de chaque échantillon pipeté dans une fiole de $100 \mathrm{ml}$, il a été ajouté $25 \mathrm{ml}$ de la solution aqueuse d'acide oxalique à $4 \%$ et $2 \mathrm{ml}$ de la solution aqueuse de 2,6-dichloroindophénol à $0,5 \%$. Au bout de 5 minutes, l'acide ascorbique à été complètement oxydé. Après l'oxydation, $10 \mathrm{mg}$ de thio-urée ont été ajoutés afin de neutraliser l'excès d'oxydant ; le mélange a été complété à $100 \mathrm{ml}$ avec de l'eau distillée. Vingt (20) $\mathrm{ml}$ du mélange obtenu ont été prélevés dans un erlenmeyer de $50 \mathrm{ml}$ avec ajout de $4 \mathrm{ml}$ de la solution de 2,4dinitrophenylhydrazine à $2 \%$ et $4 \mathrm{ml}$ d'acide sulfurique $(70 \%)$.

Le contenu de l'erlenmeyer a été transféré dans une ampoule à décanter de 500 $\mathrm{ml}$ où l'hydrazone a été extrait avec $3 \times 6 \mathrm{ml}$ du mélange acétate d'éthyle-acide acétique dans les proportions de 98:2. Les hydrazones extraits de chaque échantillon ont été rassemblés et complétés à $20 \mathrm{ml}$ avec le mélange acétate d'éthyle-acide acétique. Ces hydrazones deviennent les solutions à analyser à la CCMHP-densitométrique. Cette dérivation a été appliquée aussi bien aux échantillons qu'à la solution d'acide ascorbique standard. Environ un (1) $\mathrm{ml}$ de l'hydrazone de chaque échantillon et de la solution standard a été introduit dans les vials du déposeur qui a effectué les dépôts sur une phase stationnaire constituée de plaque HPTLC Silica gel $60 \mathrm{~F}_{254 \mathrm{~S}} 20$ x 10 (Merck $^{\circledR}$ ) sur support de verre. Les volumes déposés sont $1 ; 3$ et $5 \mu \mathrm{l}$ pour le standard et $15 \mu \mathrm{l}$ pour les échantillons. Les dépôts sont des bandes de $6 \mathrm{~mm}$ de longueur espacées de 11,2 $\mathrm{mm}$. Après les dépôts, la plaque a été retirée, séchée à l'air pendant 10 minutes et développée dans une cuve conventionnelle contenant la phase mobile constituée du mélange acétate d'éthyle - chloroforme dans les proportions de 1:1. Le temps de saturation de la plaque à été de 20 minutes. La distance de migration est $80 \mathrm{~mm}$. A la fin, la plaque a été retirée de la cuve, séchée, observée aux rayons UV et placée dans le densitomètre
Camag TLC Scanner III. La lecture de la plaque a été réalisée à $360 \mathrm{~nm}$.

\section{Détermination des composés phytochimiques}

Les composés phytochimiques ont été déterminés par un criblage phytochimique. Il s'agit d'une analyse qualitative basée sur des réactions de coloration et/ou de précipitation, réalisées sur les feuilles séchées et broyées selon la méthodologie décrite par Houghton et Raman (1998).

\section{Evaluation de la toxicité des feuilles}

L'évaluation de la toxicité des feuilles a été réalisée avec le test de survie des larves de crevettes saumure (Artemia salina LEACH) en présence de la solution à tester. C'est une méthode de bio-essai d'évaluation préliminaire de toxicité dans la recherche de produits actifs naturels. La solution à tester a été réalisée avec les feuilles à analyser. La toxicité est évaluée à partir de la concentration létale $\left(\mathrm{CL}_{50}\right)$ dont la valeur est déterminée en se servant du tableau de correspondance de Mousseux (1995) (Tableau 3).

\section{Analyse statistique des données}

Les données des analyses physicochimiques ont été d'abord saisies dans Excel 2007 et analysées avec le logiciel SPSS v 16.0. La comparaison des moyennes a été effectuée par (ANOVA) par le test de Student. Le logiciel Minitab.14 nous a permis de savoir s'il y a une différence significative entre les différents composés évalués dans les feuilles traitées avant et après emballage. Les résultats obtenus du dosage de la vitamine ont été traités et analysés avec le logiciel winCATS 1.2.4. Les données dose-réponse obtenues au cours du test de toxicité ont été transformées par logarithme afin de déterminer la Concentration létale 50 par régression linéaire conformément à la méthode décrite par Mousseux (1995). La toxicité a été évaluée à partir des valeurs $\mathrm{CL}_{50}$ en utilisant le tableau de correspondance établi par Mousseux (1995). 
Tableau 1 : Composition des solutions d'étalonnage de biuret.

\begin{tabular}{lllllll}
\hline $\mathbf{N}^{\circ}$ tube & $\mathbf{0}$ & $\mathbf{1}$ & $\mathbf{2}$ & $\mathbf{3}$ & $\mathbf{4}$ & $\mathbf{5}$ \\
\hline Etalon $(\mathrm{ml})$ & 0 & 0,2 & 0,4 & 0,6 & 0,8 & 1 \\
Eau distillée (ml) & 1 & 0,8 & 0,6 & 0,4 & 0,2 & 0 \\
Volume du réactif de Gornall $(\mathrm{ml})$ & 3 & 3 & 3 & 3 & 3 & 3 \\
\hline
\end{tabular}

Tableau 2 : Quantification des protéines dans les solutions testées.

\begin{tabular}{lc}
\hline $\mathbf{C L}_{50}$ & Toxicité \\
\hline $\mathrm{CL}_{50} \geq 100 \mu \mathrm{g} / \mathrm{ml}$ ou $0,1 \mathrm{mg} / \mathrm{ml}$ & - \\
$100 \mu \mathrm{g} / \mathrm{ml}>\mathrm{CL}_{50} \geq 50 \mu \mathrm{g} / \mathrm{ml}$ ou $0,05 \mathrm{mg} / \mathrm{ml}$ & + \\
$50 \mu \mathrm{g} / \mathrm{ml}>\mathrm{CL}_{50} \geq 10 \mu \mathrm{g} / \mathrm{ml}$ ou $0,01 \mathrm{mg} / \mathrm{ml}$ & ++ \\
$\mathrm{CL}_{50}<10 \mu \mathrm{g} / \mathrm{ml}$ & +++ \\
\hline - non toxique $;+$ faible toxicité $;++$ toxicité modéré $;+++$ forte toxicité
\end{tabular}

Tableau 3 : Tableau de correspondance pour la détermination de la $\mathrm{CL}_{50}$.

\begin{tabular}{lll}
\hline $\mathbf{N}^{\circ}$ tube & $\mathbf{6}$ & $\mathbf{7}$ \\
\hline Eau distillée $(\mathrm{ml})$ & 0,5 & 0,5 \\
Volume du réactif de Gornall $(\mathrm{ml})$ & 3 & 3 \\
Volume de solution protéique $(\mathrm{ml})$ & 0,5 & 0,5 \\
\hline
\end{tabular}

\section{RESULTATS}

\section{Caractéristiques physico-chimiques et} nutritionnelles des emballages-feuilles

La teneur en eau de toutes les espèces de feuilles analysées augmente après trois jours de conservation (Tableau 4).

\section{Profil des composés phytochimiques identifiés \\ Les composés phytochimiques} identifiés dans les espèces de feuilles traitées avant et après leur utilisation comme emballage sont résumés dans les Tableaux 5 et 6. On s'aperçoit que quel que soit le traitement auquel les feuilles ont été soumises avant et/ou après avoir été utilisées comme emballages, les quatre espèces possèdent en commun les grands groupes de composés chimiques à savoir : les tanins catéchiques, les anthocyanes, les composés réducteurs, exceptés les flavonoïdes et les coumarines qui sont retrouvés uniquement dans les feuilles de Tectona grandis et Musa sapientum (Tableaux 5 et 6$)$.

\section{Toxicité des feuilles}

Les Figures 1a, b, c et d traduisent la variation du nombre de larves mortes en fonction de la concentration de l'extrait testé. L'analyse des résultats montre que les larves de crevettes sont sensibles aux extraits testés selon la relation dose-réponse.

La concentration de $50 \%$ de survie des larves a été déterminée et les résultats sont consignés dans le Tableau 7. 
Tableau 4 : Teneur en eau, en protéines et en lipides totaux avant et après emballage $(\mathrm{g} / 100)$, en fer $(\mathrm{mg} / 100 \mathrm{~g})$ des espèces, en vitamine $\mathrm{C}$ en $\mathrm{mg}$ pour $100 \mathrm{~g}$ de feuilles fraîches.

\begin{tabular}{lcccc}
\hline & \multicolumn{4}{c}{ Espèces de feuilles végétales servant d'emballages } \\
\cline { 2 - 5 } Constituants & Tectona grandis & Musa sapientum & Thalia geniculata & Manihot esculenta \\
\hline Eau $_{1}$ & $59,31 \pm 0,26 \mathrm{a}$ & $66,65 \pm 1,47 \mathrm{a}$ & $70,95 \pm 1,24 \mathrm{a}$ & $62,32 \pm 0,30 \mathrm{a}$ \\
Eau $_{2}$ & $60,90 \pm 1,04 \mathrm{~b}$ & $70,84 \pm 0,63 \mathrm{~b}$ & $72,34 \pm 0,64 \mathrm{~b}$ & $74,10 \pm 0,13 \mathrm{~b}$ \\
Protéines totaux $_{1}$ & $6,08 \pm 1,13 \mathrm{a}$ & $16,01 \pm 1,47 \mathrm{a}$ & $12,50 \pm 0,70 \mathrm{a}$ & $13,45 \pm 0,47 \mathrm{a}$ \\
Protéines totaux $_{2}$ & $0,83 \pm 1,13 \mathrm{~b}$ & $9,75 \pm 1,90 \mathrm{~b}$ & $7,30 \pm 0,18 \mathrm{~b}$ & $8,61 \pm 0,23 \mathrm{~b}$ \\
Lipides totaux $_{1}$ & $4,65 \pm 0,46 \mathrm{a}$ & $4,34 \pm 0,25 \mathrm{a}$ & $4,62 \pm 0,29 \mathrm{a}$ & $2,01 \pm 0,01 \mathrm{a}$ \\
Lipides totaux $_{2}$ & $2,50 \pm 0,35 \mathrm{~b}$ & $2,05 \pm 0,21 \mathrm{~b}$ & $2,21 \pm 0,12 \mathrm{~b}$ & $0,95 \pm 0,02 \mathrm{~b}$ \\
Fer $_{1}$ & $4,44 \pm 0,15 \mathrm{a}$ & $0,82 \pm 0,14 \mathrm{a}$ & $1,74 \pm 0,02 \mathrm{a}$ & $8,44 \pm 0,14 \mathrm{a}$ \\
Fer $_{2}$ & $2,35 \pm 0,14 \mathrm{~b}$ & $0,81 \pm 0,01 \mathrm{a}$ & $1,73 \pm 0,01 \mathrm{a}$ & $5,32 \pm 0,14 \mathrm{~b}$ \\
VitamineC $_{1}$ & - & - & $397,02 \pm 0,10 \mathrm{a}$ & - \\
VitamineC $_{2}$ & - & - & $126,05 \pm 0,02 \mathrm{~b}$ & - \\
\hline Les indices & et & représentent respectivement les paramètres étudiés avant et après emballage. Les valeurs portant les lettres \\
différentes sur la même colonne pour un paramètre donné sont significativement différentes au seuil de 5\%.
\end{tabular}

Tableau 5 : Profil des Composés phytochimiques identifiés dans les emballages-feuilles traitées avant leur utilisation comme emballage.

\begin{tabular}{|c|c|c|c|c|}
\hline \multirow[t]{2}{*}{ Composés chimiques avant emballage } & \multicolumn{4}{|c|}{ Espèces de feuilles végétales étudiées } \\
\hline & $\begin{array}{l}\text { Tectona } \\
\text { grandis }\end{array}$ & $\begin{array}{c}\text { Musa } \\
\text { sapientum }\end{array}$ & $\begin{array}{c}\text { Thalia } \\
\text { geniculata }\end{array}$ & $\begin{array}{c}\text { Manihot } \\
\text { Esculenta }\end{array}$ \\
\hline Composés réducteurs & ++ & ++ & ++ & ++ \\
\hline Anthocyanes & ++ & + & ++ & ++ \\
\hline Mucilages & + & ++ & ++ & ++ \\
\hline Tanins catéchiques & ++ & + & ++ & ++ \\
\hline Flavonoïdes & ++ & + & - & - \\
\hline Coumarines & + & + & - & - \\
\hline Leuco-anthocyanes & + & - & - & - \\
\hline Alcaloïdes & - & - & - & - \\
\hline Tanins galliques & - & - & - & - \\
\hline Dérivés quinoniques & - & - & - & - \\
\hline Saponosides & - & - & - & - \\
\hline Stéroïdes & - & - & - & - \\
\hline Terpènes & - & - & - & - \\
\hline Recherche des dérivés cyanogéniques & - & - & - & - \\
\hline Dérivés anthracéniques C-hétérosides & - & - & - & - \\
\hline O-hétérosides & - & - & - & - \\
\hline Hétérosides cardiotoniques & - & - & - & - \\
\hline
\end{tabular}


Tableau 6: Profil des Composés phytochimiques identifiés dans les emballages-feuilles traitées après leur utilisation comme emballage.

\begin{tabular}{|c|c|c|c|c|}
\hline \multirow[b]{2}{*}{ Composés chimiques avant emballage } & \multicolumn{4}{|c|}{ Espèces de feuilles végétales analysées } \\
\hline & $\begin{array}{l}\text { Tectona } \\
\text { grandis }\end{array}$ & $\begin{array}{c}\text { Musa } \\
\text { sapientum }\end{array}$ & $\begin{array}{c}\text { Thalia } \\
\text { geniculata }\end{array}$ & $\begin{array}{c}\text { Manihot } \\
\text { Esculenta }\end{array}$ \\
\hline Composés réducteurs & ++ & ++ & ++ & ++ \\
\hline Mucilages & + & ++ & ++ & ++ \\
\hline Anthocyanes & + & + & + & + \\
\hline Tanins catéchiques & + & + & + & + \\
\hline Flavonoïdes & + & + & - & - \\
\hline Coumarines & + & + & - & - \\
\hline Leuco-anthocyanes & + & - & - & - \\
\hline Alcaloïdes & - & - & - & - \\
\hline Tanins galliques & - & - & - & - \\
\hline Dérivés quinoniques & - & - & - & - \\
\hline Saponosides & - & - & - & - \\
\hline Stéroïdes & - & - & - & - \\
\hline Terpènes & - & - & - & - \\
\hline Recherche des dérivés cyanogéniques & - & - & - & - \\
\hline Dérivés anthracéniques C-hétérosides & - & - & - & - \\
\hline O-hétérosides & - & - & - & - \\
\hline Hétérosides cardiotoniques & - & - & - & - \\
\hline
\end{tabular}

Tableau 7 : Valeur de la $\mathrm{CL}_{50}$ des extraits des emballages-feuilles testés.

\begin{tabular}{lc}
\hline Extraits des feuilles & $\mathbf{C L}_{\mathbf{5 0}}(\mathbf{m g} / \mathbf{m l})$ \\
\hline Extrait de Tectona grandis & 0,78 \\
Extrait de Musa sapientum & 0,19 \\
Extrait de Thalia geniculata & 1,56 \\
Extrait de Manihot esculenta & 0,78 \\
\hline
\end{tabular}

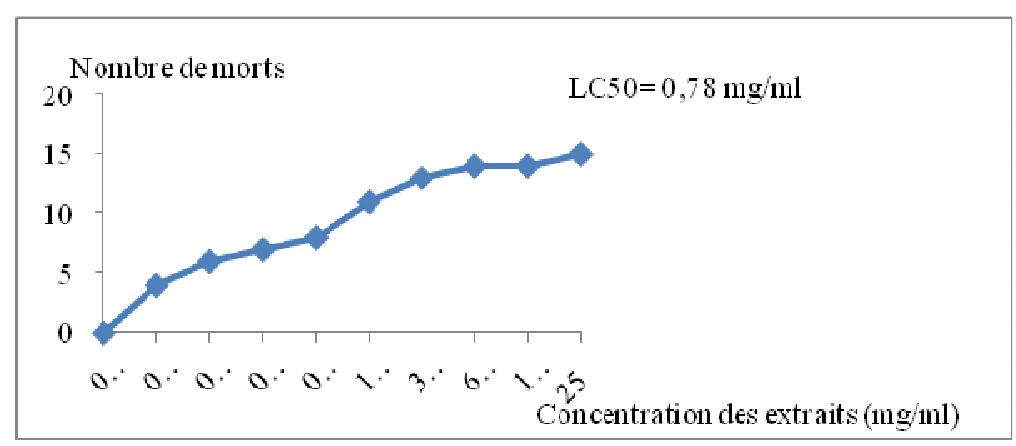

a) Tectona grandis 


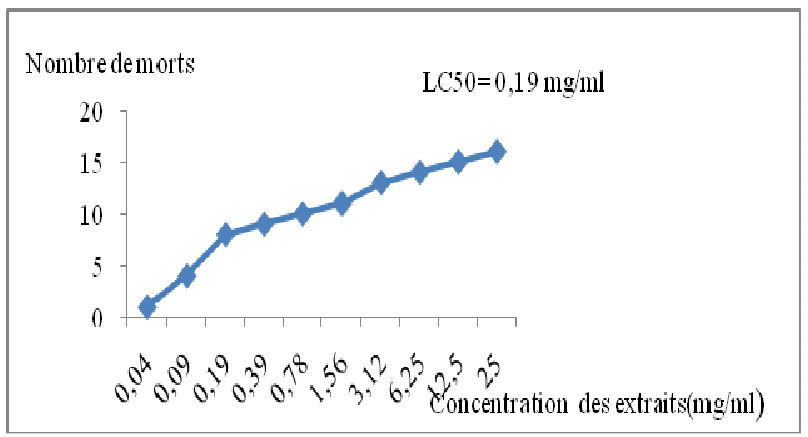

b) Musa sapientum

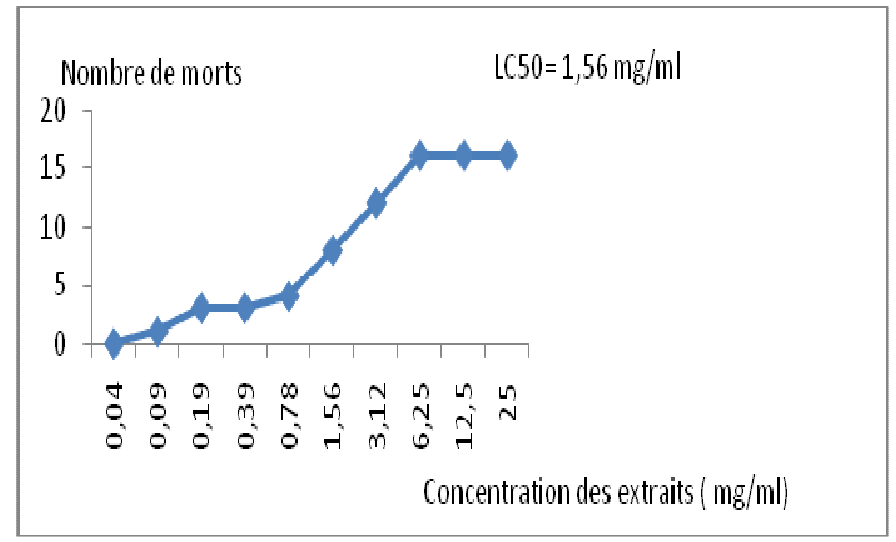

c) Thalia geniculata

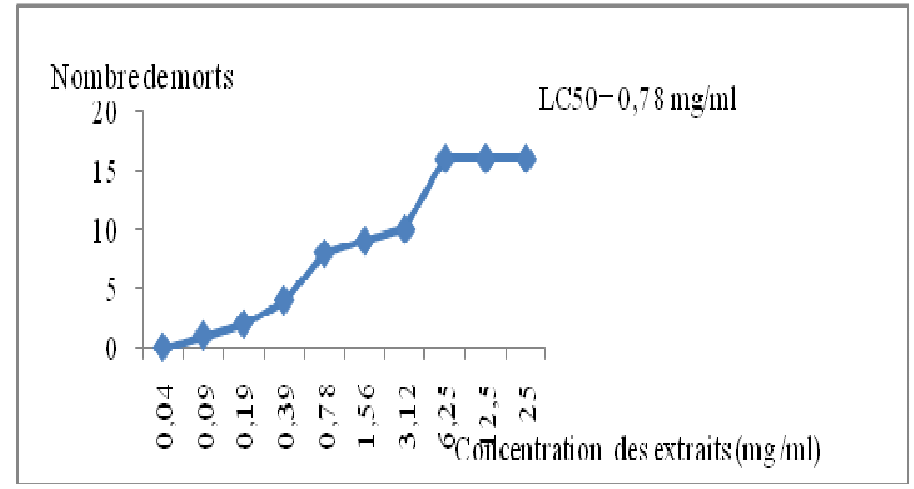

d-) Manihot esculenta

Figure 1 : Sensibilité des larves de crevettes aux extraits des espèces de feuilles végétales analysées.

\section{DISCUSSION}

L'augmentation de la teneur en eau pourrait se justifier par deux phénomènes : le premier phénomène serait lié au fait que la dernière couche des feuilles qui est en contact direct avec le produit emballé aurait pris d'humidité du produit frais emballé par le mécanisme d'absorption au cours duquel il y a eu probablement un transfert d'eau. Le deuxième phénomène serait lié au fait que 
l'emballage a engendré une augmentation de la température a notamment au niveau de la dernière couche en contact avec le produit qui par suite d'une condensation, aurait engendré une augmentation du taux d'humidité aussi bien du produit que de celui des emballagesfeuilles.

Par ailleurs, après la cuisson à la vapeur, une opération qui est réalisée au cours de la fabrication des boules d'akassa, la teneur en eau aurait augmenté considérablement dans les feuilles de Manihot esculenta. La perméabilité à la vapeur d'eau des emballages biodégradables a été rapportée par Rabetafika et Paquot (2006). Cette forte teneur en eau des feuilles les rend sensibles à la prolifération des micro-organismes qui peuvent non seulement les détériorer en quelques jours, mais peuvent être également transférés aux produits emballés. Etant donné que ce sont des emballages qui sont utilisés, aussi bien pour le conditionnement que pour la consommation directe. Une telle prolifération microbienne n'est pas favorable pour une meilleure stabilité du produit et est préjudiciable à la santé des consommateurs, d'où l'importance d'observation des bonnes conditions de conservation ou de consommation des produits emballés comme suggéré par Wagner (2004), Tossou et al. (2007), Adegunloye et al. (2006) et Adejumo et Ola (2008).

Une diminution significative de la teneur en lipides totaux est observée au niveau de toutes les espèces de feuilles analysées après emballage. Cette diminution pourrait s'expliquer par une probable migration des constituants chimiques de l'emballage vers l'aliment au cours de la durée de conservation. Il a été rapporté que la migration des constituants chimiques de l'emballage augmenta en fonction de la durée de conservation (Felgenbaum, 1996).

Toutes les espèces contiennent du fer, notamment Tectona grandis et Manihot esculenta avec des teneurs très proches des valeurs recommandées. En effet, les valeurs journalières normatives recommandées sont respectivement de $10 \mathrm{mg}$ pour les enfants de 3 à 12 ans, $28 \mathrm{mg}$ pour les adolescents de 12 à
20 ans et $9 \mathrm{mg}$ pour les adultes modérément actifs. Cependant, une diminution significative $\mathrm{du}$ fer dans les espèces mentionnées a été observée après emballage, notamment au cours de la conservation. Ces espèces peuvent être recommandées pour le traitement des jeunes enfants anémiés (Nacoulma, 1996a ; Onzo et al., 2013).

Aucune présence de vitamine C n'a été observée au niveau de trois sur les quatre espèces de feuilles étudiées. En revanche, une teneur en vitamine $\mathrm{C}$ relativement élevée a été observée dans les espèces de feuilles de Manihot esculenta avant l'emballage. En effet, il a été rapporté que les feuilles de Manihot esculenta sont généralement riches en vitamine C (Rakipov, 1985), ce qui justifie leur utilisation dans l'alimentation humaine où elles sont directement consommées comme légume-feuilles après avoir été utilisées comme emballage.

Dans de nombreux ménages traditionnels au Bénin, les feuilles de Manihot esculenta ayant servi à emballer les produits sont, par la suite, utilisées comme légumefeuilles pour la préparation de sauces. La vitamine $\mathrm{C}$ est un anti-oxydant qui participe à l'absorption du fer et aide à la lutte contre les infections. Sa consommation prévient également contre le scorbut. L'akassa cru est d'abord emballé dans les feuilles de Manihot esculenta avant d'être soumis à une cuisson à vapeur. La diminution drastique de la teneur en vitamine $\mathrm{C}$ dans les feuilles après cuisson pourrait être donc liée à une perte du fait de la cuisson à la vapeur effectuée au cours du procédé de préparation de l'akassa. Il faut donc trouver des approches de solutions susceptibles de préserver au maximum la teneur en vitamine $\mathrm{C}$ dans cette espèce de feuilles pour son double utilisation comme emballage et comme légume-feuilles. Cette double utilisation a un avantage comparatif du point de vue nutritif.

S'agissant du profil des composés phytochimiques, il a été rapporté que les anthocyanes sont des pigments hydrosolubles responsables de la coloration des feuilles (Nacoulma, 1996a). Cette coloration naturelle 
est instable en milieu aqueux et varie avec le $\mathrm{pH}$ et la chaleur (Nacoulma, 1996b). La présence des tanins dans les espèces présente un avantage particulier étant donné que les tanins présentent des activités anticryptococcuques, antivirales, antiinflammatoires, antimutagène, anti-hypertensives, antitumorales et anti-diarrhéiques relativement élevées (Kolodzeiej et al., 1999; Bruneton, 1999 ; Feldman et al.,1999 ).

En outre, on reconnaît aux flavonoïdes des activités antivirales, antitumorales, antiinflammatoires et antiallergiques (Nacoulma, 1996b). De plus, les coumarines présentent des propriétés anti-coagulantes, antioxydantes, anti-inflammatoires (Nacoulma, 1996b).

Vu les propriétés pharmacologiques de ces différents groupes chimiques retrouvés dans ces feuilles, leur utilisation comme à la fois emballage et légumes-feuilles pourrait aider à soigner diverses maladies, à condition que ces différents composés puissent être transmis en dose effectrice dans les aliments emballés. En outre, l'absence des composés toxiques comme les hétérosides cardiotoniques, les dérivés cyanogéniques, les dérivés quinoniques qu'on retrouve généralement dans les mêmes groupes garantit une certaine sécurité sanitaire des feuilles, notamment les espèces utilisées comme légume-feuilles telles que Thalia geniculata (Nacoulma, 1996 a, b) et Manihot esculenta (Adjanohoun et al., 1989). On peut, certes, redouter les variétés de feuilles de Manihot esculenta généralement plus riches en composés cyanogéniques qui sont des précurseurs d'acide cyanhydrique qui est un produit toxique (Wagner, 2004). Mais avec la cuisson à la vapeur à laquelle les boules d'akassa sont soumises, la présence d'acide cyanhydrique est très peu probable. Mais il est indispensable qu'un dosage des différents composés précédemment mentionnés, notamment, l'acide cyanhydrique soit réalisé aussi bien sur les emballages-feuilles que sur les produits emballés pour permettre d'avoir une compréhension précise de leur transfert ou non.
Par ailleurs le test de toxicité a montré que les extraits testés ne présentent pas de toxicité à la dose appliquée (Tableau 7). Par conséquent, en considérant la corrélation entre la cytotoxicité sur les larves de crevettes et sur les cellules 9 PS et $9 \mathrm{~KB}$ (carcinome nasopharyngien humain) (Pelka et al., 2000) d'une part, les cellules A-549 du carcinome pulmonaire et les cellules HT-29 du côlon (Carballo et al., 2002), d'autre part, on peut dire que les extraits testés ne présentent pas d'activité cytotoxique et les espèces dont ils proviennent peuvent donc être utilisées comme emballages alimentaires sans aucun risque de toxicité.

\section{Conclusion}

Les feuilles étudiées constituent des sources importantes de protéines, de lipides et de fer. Elles renferment également des molécules chimiques telles que les tannins, les composés réducteurs, les anthocyanes, les flavonoïdes, les mucilages et les coumarines qui sont des principes actifs utilisés dans le traitement de certaines pathologies humaines. Ces différents composés sont transférés des feuilles vers les produits emballés. La consommation des ces espèces de feuilles présente donc un intérêt du point de vue nutritif, surtout qu'elles ne présentent aucun risque de toxicité. Il est souhaitable que ces différentes espèces de feuilles soient domestiquées afin de garantir leur disponibilité à plein temps.

\section{REMERCIEMENTS}

Tous nos remerciements à l'Union Economique Monétaire Ouest Africaine (UEMOA) pour l'appui financier. Nos remerciements vont également au centre de Biométrie et d'Informatique Générale de la Faculté des Sciences Agronomiques de l'Université d'Abomey-Calavi (CBIG/FSA/ UAC) pour l'appui technique.

\section{REFERENCES}

Adegunloye DV, Agarry OO, Adebolu TT, Adetuyi FC. 2006. Effect of leafpackaging on the microbiological 
assessment of some food items. African Journal of Biotechnology, 5: 445-447.

Adejumo BA, Ola FA. 2008. The appraisal of local food packaging materials in Nigeria. Continental J. Engineering Sciences, 3:13-20

Adjanohoun EJ, Adjakidjè V, Ahyi MRA, Aké Assi L, Akoègninou A, D'almeda J, Apovo F, Boukef K, Chadaré M, Cusset G, Dramane K, Eymé J, Gassita JN, GbaguidiN, Goudoté E, Guinko S, Houngn on P, Issa Lo, Kéita A, Kiniffo HV, Koné-Bamba D, Musampa Nséyya A, Saadou M, Sodogandji T, De Souza S, Tchabi A, Zinsou Dossa G, Zohoun T. 1989. Contribution aux Etudes Ethnobotaniques et Floristiques en République $d u$ Bénin. Agence de Coopération Culturelle et Technique : Bénin ; 895.

AFNOR (Association Française de Normalisation). 1974. Emballages, matières en feuille, détermination du coefficient de transfert de vapeur d'eau. Association Française de Normalisation, $30 \mathrm{p}$.

AFNOR (Association Française de Normalisation).1986. Emballages en papier, carton, bois, matières plastiques. Association Française de Normalisation $228 \mathrm{p}$.

AOAC (Association of Official Analytical Chemists). 1984. Official methods of Analysis. $\left(14^{\text {th }}\right.$ edn). Association of Official Analytical Chemists, Minnesota: USA.

Boko M, Heideveld A, Elsen A. 1997. Introducing Leaf Packaging in the Netherlands, A UNB/ UvA / UNEPWG-SPD collaborative project, National University Benin (UNB).

Bruneton J. 1999. Les Tannins; Ed Médicales Internationales. Tec \& Doc : Paris ; 369404.

Carballo JL, Hernández-Inda ZL, Pérez P, García-Grávalos $\quad$ MD. 2002. Comparaison between to brine shrimp assays to detect in-vitro cytotoxicity in marine natural products. BMC Biotechnology, 2: 17.

Cavassilas M. 2006. Clés et Codes du Packaging: Sémiotique Appliqué. Edition Hermes Sciences et Publications ; 54.

Codjia RP. 2000. La prolifération des déchets plastiques à Cotonou : Effet sur la santé et sur le développement durable, Mémoire de DEA, UNB, 54p.

Feldman KS, Sahasrabudhe K, Smith R S, Scheuchenzuber WJ. 1999. Immunostimulation by plant polyphenols. A relationship between tumor necrosis factor-alpha production and tannin structure. Biorganic and Medical Chemistry Letters, 9(7): 985990.

Felgenbaum A. 1996. Evaluation de la Migration des Matériaux Plastiques au Contact des Aliments, par des Méthodes Alternatives dans l'emballage des Denrées Alimentaires de Grande consommation. Edition Lavoisier ; 92.

Gornall AG, Bardwill CJ, David M. 1949. Determination of serum protein by means of biuret reaction. Journal of Biochemy, 177: 751-766.

Grubben GJH. 1975. La culture de l'Amaranthe, légume-feuilles tropical, avec référence spéciale au SudDahomey. Mededelingen Landbouwhogeschool Wageningen: Landbouwhogeschool Netherlands; 223.

Kolodziej KO, Latte KP, Ferreira D. 1999. Evaluation of the antimicrobial potency of tannins and related compounds using the microdilution both method. Planta Medica, 65(5): 444-446.

Houghton PJ, Raman A. 1998. Laboratory Handbook for the Fractionation of Natural Extracts. Ed. Chapman and Hall: New York; 208.

Hounhouigan DJ. 2000. Matières végétales au Bénin. Un potentiel d'emballages biodégradables, In. Bulletin du Réseau TPA. Les Emballages Alimentaires, 17: 29-41. 
Karel M, Heidelbaugh ND. 1975. Effects of Packaging and Nutrients in Nutritional Evaluation of Food Processing (2nd edn). AVI Publishing Co. Westport, Connecticus.

Kazazian T. 1995. Le Cycle de l'Emballage: Le Conditionnement de Qualité Environnementale. Ed. Masson: Paris ; 95.

Kerharo J, Adam JG. 1974. La Pharmacopée Sénégalaise Traditionnelle. Plantes Médicinales et Toxiques. Editions Vigot Frères : Paris; 1011.

Mousseux M. 1995. Test de toxicité sur les larves d'Artemia salina, entretien d'un élevage de balanes, Rapport de stage de 2ème année, Deust aquaculture ; Centre Universitaire de Nouvelle Calédonie, France.

Nacoulma O. 1996a. Plantes médicinales et pratiques médicinales traditionnelles au Burkina Faso. Cas du plateau central, Tome 1, Thèse de Doctorat d'Etat ès Sciences Naturelles, Université de Ouagadougou, 315p.

Nacoulma O. 1996b. Plantes médicinales et pratiques médicinales traditionnelles au Burkina Faso. Cas du plateau central, Tome 2 Annexes; Thèse de Doctorat d'Etat ès Sciences Naturelles, Université de Ouagadougou, 259p.

Onzo CF, Azokpota P, Akissoe N, Agbani P. 2013. Biodiversité des feuilles végétales utilisées comme emballages dans l'artisanat agroalimentaire au Sud du Bénin. Journal of Applied Biosciences, 72: 5810- 5821 .
Pelka M, Danzl C, Distler W, Petschelt A. 2000. A new screening test toxicity testing of dental materials. J. Dent, 28: 341-345.

Pothet JP. 2003. Emballage et conditionnement - Marketing Techniques - Mise en cuvre - Qualité Réglementation. Collection Les Référentiels Dunod : Paris ; 420.

Rabetafika H, Paquot D. 2006. Les polymères issus du végétal : matériaux à propriétés spécifiques pour des applications ciblées en industrie plastique. Biotechnologie, Agronomie, Sociologie, Environnement, http://babordplus.univ-bordeaux.fr/ notice.php?q=issn:(1370-6233)\&fq= typedoc:(s)

Rakipov N. 1987. Biochimie des Cultures Tropicales. Editions Mir Moscou ; 335p.

Reyne M. 1991. Les Plastiques dans l'Emballage: Polymères, Technologie de Mise en Forme, Recyclage. Ed. Hermès Science Publications : Oxford ; 92p.

Tossou R, Vodouhè S, Assogba M, Fanou J, Kouévi A. 2007. Dynamique d'urbanisation à Abomey-Bohicon (Bénin): opportunités et contraintes pour la filière maïs, UAC, AbomeyCalavi FSA, document de travail Ecocité, 44p.

Wagner T. 2004. Le Lio, un Produit de Terroir. Analyse des Systèmes de Production du Lio à Abomey et Bohicon, Johannes Gutemberg Universitat : Johannes Gutemberg; 51p. 